\title{
USING 3D MODELLING TECHNIQUES FOR SURVEYS IN TWO DIMENSIONAL SCALE MODEL TESTS OF BREAKWATERS
}

Rute LEMOS ${ }^{*}$, Maria HENRIQUES ${ }^{1}$, António MURALHA ${ }^{1}$, Ricardo JÓNATAS' ${ }^{1}$, Conceição FORTES ${ }^{1}$, Rui CAPITÃO $^{1}$

\section{Abstract}

This paper focuses on a study of different methodologies for surveying two-dimensional scale models of rubble mound breakwaters.

Tests were conducted in one of the LNEC's irregular wave flumes and involved the use of four different methodologies using photogrammetric and 3D scanning techniques.

This paper describes the materials and methods used during the experiments, as well as the results obtained.

The data analysis contains the profiles and comparison of the meshes used in each of the techniques, in order to evaluate their application in scale model tests of rubble mound breakwaters. Also, the specific experimental conditions needed for using each of these techniques were investigated.
Address

1 Laboratório Nacional de Engenharia Civil, Hydraulics Department

* Corresponding author: rlemos@lnec.pt

\section{Key words}

- Maritime structures,

- Scale model tests,

- Damage evolution,

- 3D surface models,

- Photogrammetry.

\section{INTRODUCTION}

Due to its extensive coastal zone, Portugal owns a number of relevant maritime structures such as artificial harbours protected by breakwaters. Indeed, breakwaters are structures built to create sufficiently calm waters for safe mooring and loading operations, the handling of ships, and protection of harbour facilities (Fig. 1 Leixões harbour protected by its two main breakwaters (Portugal).). They usually also help to control sedimentation by guiding currents or protecting coastlines against the action of abnormal wave activity, such as tsunamis.

In Portugal, rubble-mound breakwaters with a armor layer usually composed of rock or artificial armor units are the most common kind of these structures (Figure 2).

Prior to its construction, the design of most of these maritime structures requires running a series of scale model tests in order to evaluate their hydraulic and structural behaviour and thereby characterize the structure's response to incident sea waves in terms of overtopping (so-called "overtopping tests") or damage in the armour

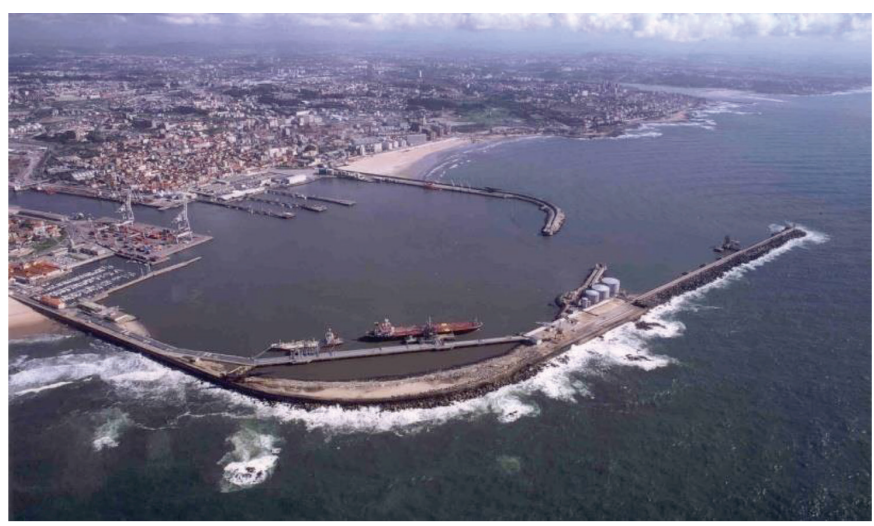

Fig. 1 Leixões harbour protected by its two main breakwaters (Portugal).

layers (so-called "stability tests").

Assessments of the evolution of damage shown by scale-model tests of rubble-mound breakwaters are traditionally conducted by 
a)

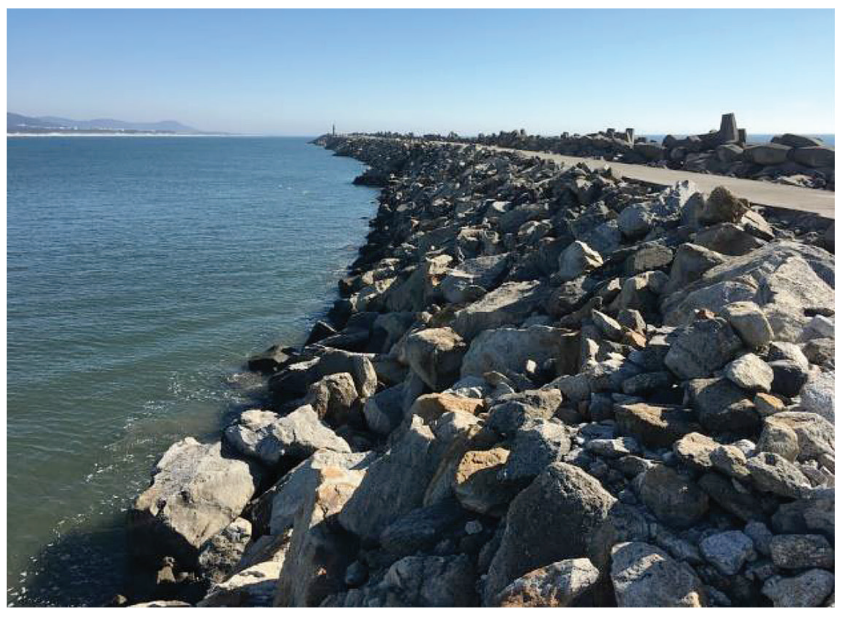

b)

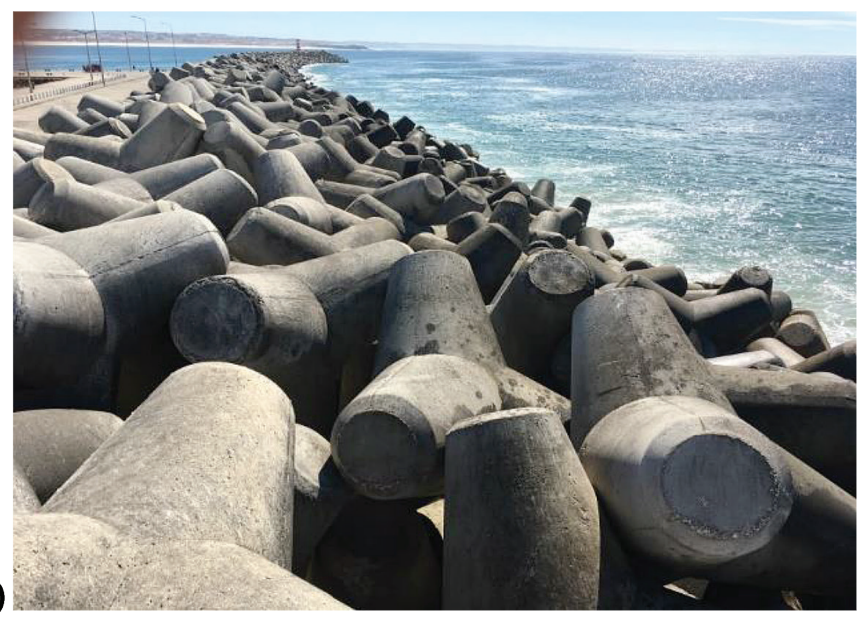

Fig. 2 Typical rubble-mound breakwaters with armour layers composed of: a) rock units, and b) artificial units.

comparing the damage in profiles which are representative of the tested section and by determining the eroded volume of the tested section between consecutive surveys. Damage to the armour layer is then characterized by parameters based either on the number of displaced armour units, as is the $N_{o d}$ parameter (van der Meer, 1988), or on dimensionless parameters based on the eroded area of a profile of the armour layer, such as $S$ (Broderick and Ahrens, 1982) or the maximum eroded depth, $E_{2 D}$ (Hofland et al., 2014, Hofland et al.,

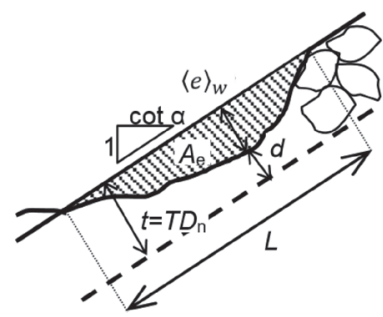

$\mathrm{S}=\mathrm{A}_{\mathrm{e}} / \mathrm{Dn}_{50}{ }^{2}$

$\langle\mathrm{e}\rangle_{\mathrm{w}}=$ eroded depth of the profile

$\mathrm{E}_{2 \mathrm{D}}=\max \left(\langle\mathrm{e}\rangle_{\mathrm{w}}\right) / \mathrm{Dn}_{50}$

$\mathrm{A}_{\mathrm{e}}=$ eroded area of the profile

$\mathrm{Dn}_{50}=$ Mean nominal rock diameter

$\mathrm{T}=$ Number of layers of armour units

$\mathrm{L}=$ Eroded length

Fig. 3 Definition of damage numbers $S$ and $E_{2 D}$ on width-averaged $2 D$ trunk profiles (Hofland et al., 2017)

\section{7) (Figure 3).}

During stability tests, the progression of damage for different incident wave conditions is assessed; They make use of visual observations, video and photographic techniques, or even mechanical profilers when profile surveys are needed.

Image processing tools based upon photogrammetric methods can be a good alternative to mechanical profilers, due to their ease of use and the required cost-effective equipment. On the other hand, although photogrammetric techniques have relevant advantages, they also have some limitations, such as the fact that their domain of application depends not only on specific experimental conditions (namely, light conditions) but also on the post-processing tools needed to construct 3D surface models, which is a key point in the success of photogrammetric techniques.

The objective of this paper is to describe and compare the use of different 3D survey techniques to create and analyse 3D surface models made of point clouds through the use of both commercial and open-source post-processing tools, combining cost-effective equipment/software.

To achieve this goal, scale model study of a section of a breakwater was set up at the Maritime Hydraulics facilities of the National Laboratory for Civil Engineering (LNEC). Four methodologies used to survey the armour layer of a rubble-mound breakwater model with digital images were applied; two DSLR cameras and a Microsoft ${ }^{\circledR}$
Kinect ${ }^{\circledR}$ sensor were used.

The first methodology was based upon scans with Time of Flight (ToF) (using the Kinect sensor). The second and third methodologies were based on photogrammetric techniques that use open-source software to process multiple photos taken from a scene to reconstruct it. The fourth methodology is a stereo-photogrammetric technique that uses photogrammetric pairs of images taken from slightly different positions. It also uses software that rectifies the distortion introduced by an air-water interface, thereby enabling the surveying of submerged scenes.

Different commercial and open-source post-processing tools were used to create the 3D surface models from point clouds, namely:

- First methodology: A proprietary Microsoft $\mathrm{f}^{\mathbb{R}}$ Kinect $^{\mathbb{B}}$ sensor application (herein called "MK methodology").

- Second methodology: A Python Photogrammetry open-source Toolbox (herein called "PP methodology");

- Third methodology: The MicMac open-source software (herein called "MM methodology");

- Fourth methodology: Ferreira software, (2006), which was specially developed for the reconstruction of submerged scenes (herein called "IS methodology").

In order to attain a clearer comparison between these tools (to better evaluate accuracy and associated errors), scale-model dimensions for all the methodologies were used in this paper. In addition, specific experimental conditions needed for using each methodology were investigated and classified.

\section{CHARACTERISATION OF THE PHYSICAL SCALE MODEL}

The tests were conducted in one of the LNEC's irregular wave flumes, i.e, the COI1 flume, a $50 \mathrm{~m}$-long wave flume with an operating width of $0.8 \mathrm{~m}$ and an operating water depth of $0.8 \mathrm{~m}$. The model tested was multi-layer rubble-mound coastal protection, consisting of a trapezoidal core covered by a $10-30 \mathrm{kN}$ rock underlayer, which was protected by a $300 \mathrm{kN}$ Antifer cubes armour layer, with a $30-60 \mathrm{kN}$ rock crest berm. The slope was approximately $3: 2$. The model being tested was $1.2 \mathrm{~m}$ long, $0.75 \mathrm{~m}$ wide and $0.59 \mathrm{~m}$ tall (Figure 4).

The equipment used for the four survey methodologies included the following (Figure 5):

- First methodology: A Kinect sensor (version 2.0), which was mounted in a camera support and able to scan the entire breakwater slope. It was connected and triggered by a laptop com- 
a)
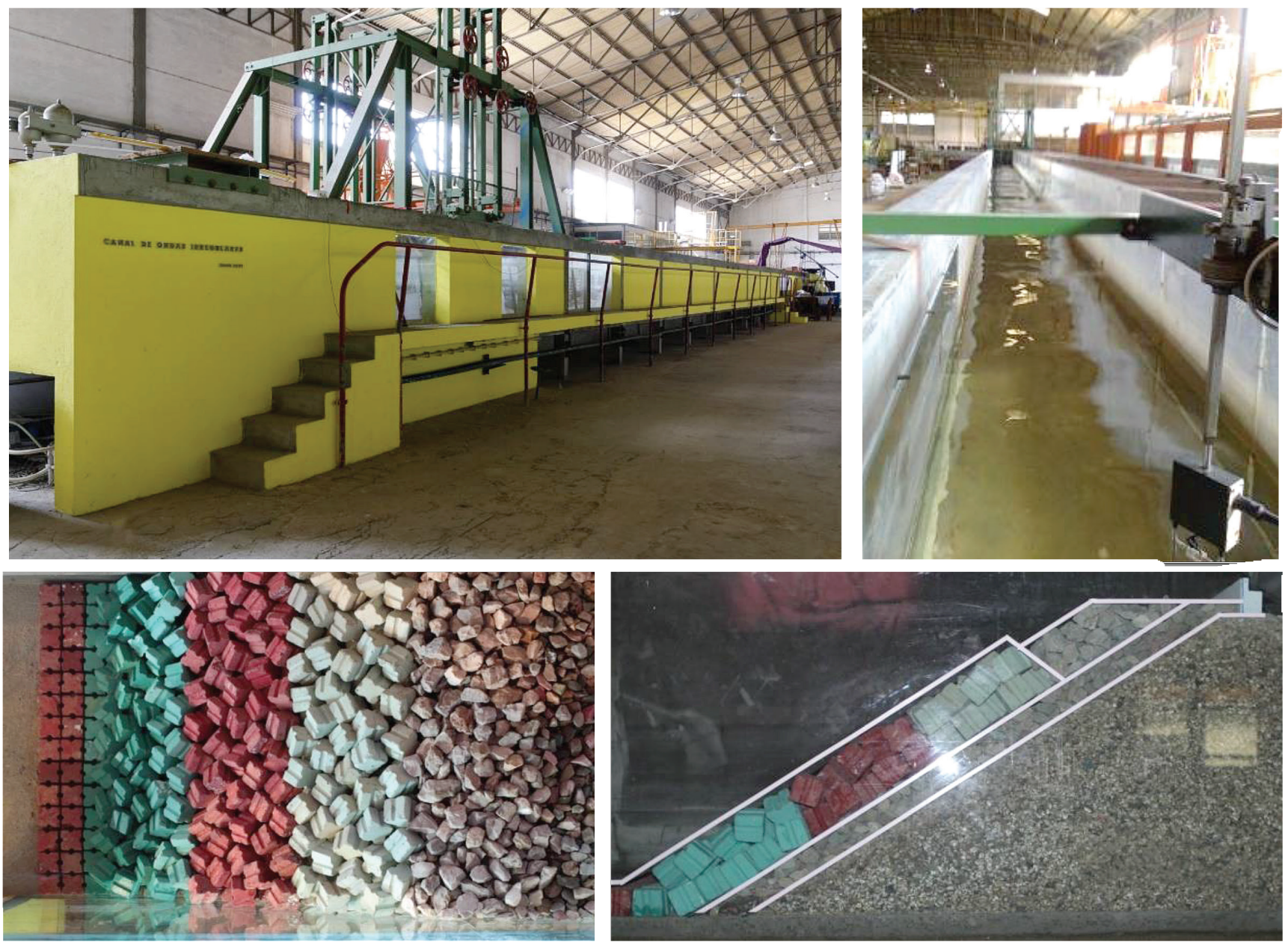

Fig. 4 Overview of the COII flume (a) and top and side views of the two-dimensional scale model (b)

puter;

- Second and third methodologies: A Canon EOS 600D digital DSLR camera with an EF $35 \mathrm{~mm} \mathrm{f} / 2$ lens was focused and triggered manually; it was able to acquire around 50 photos for the entire breakwater slope by considering an overlapping area of about $80 \%$;

- Fourth methodology: Two digital SLR cameras (Canon EOS

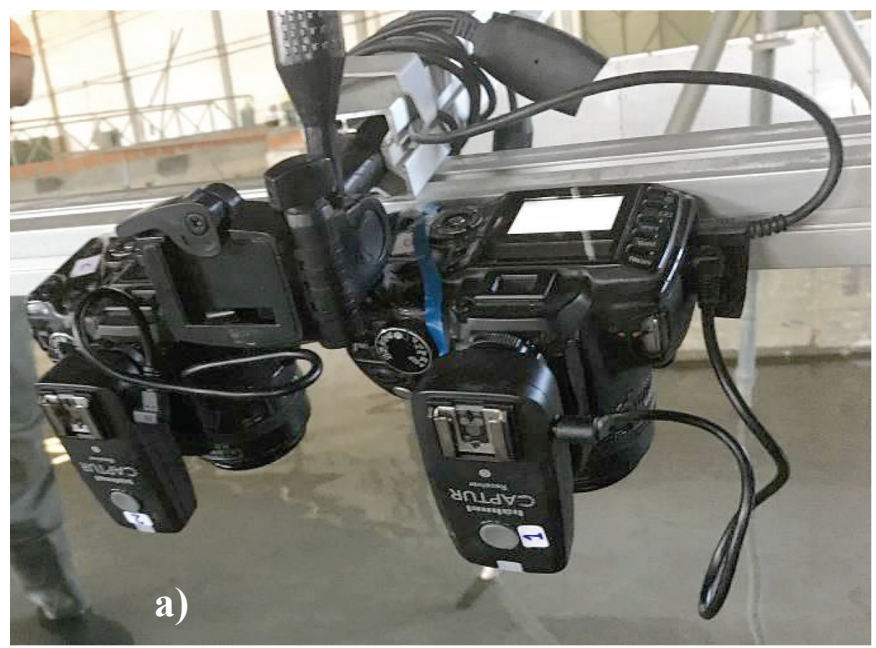

600D) fitted with fixed focal length lenses (Canon EF $35 \mathrm{~mm}$ $f / 2$ ), mounted side by side in a support structure, approximately $2 \mathrm{~m}$ above the flume and able to simultaneously capture the same scene; they were triggered from a desktop computer.

For the first three methodologies (MK, PP and MM), the reference coordinates were given by target points deployed at different points of well-known dimensions of the model (as the size of the armour units) in order to create a local referential, where the coordinates origin and direction were selected. The CloudCompare software was used to determine the 3D coordinates of the GCP.

For those methodologies, the surveys were conducted without water. For the fourth methodology (IS), the coordinate system was defined by using a pattern-chequered board with accurate dimensions

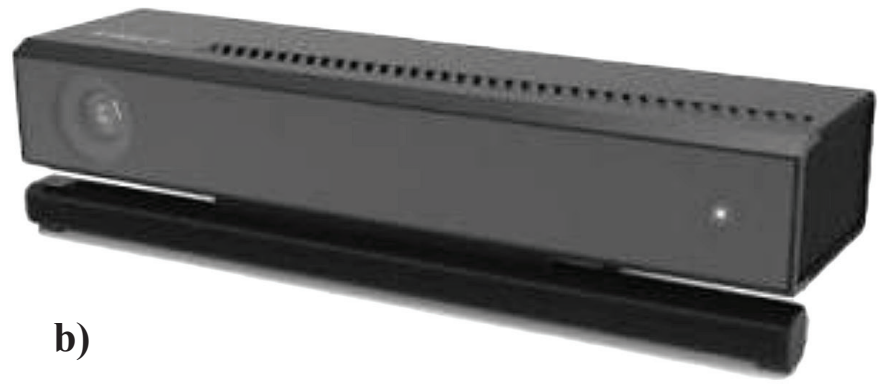

Fig. 5 Survey equipment. SLR cameras a) and Kinect 2.0 b). 

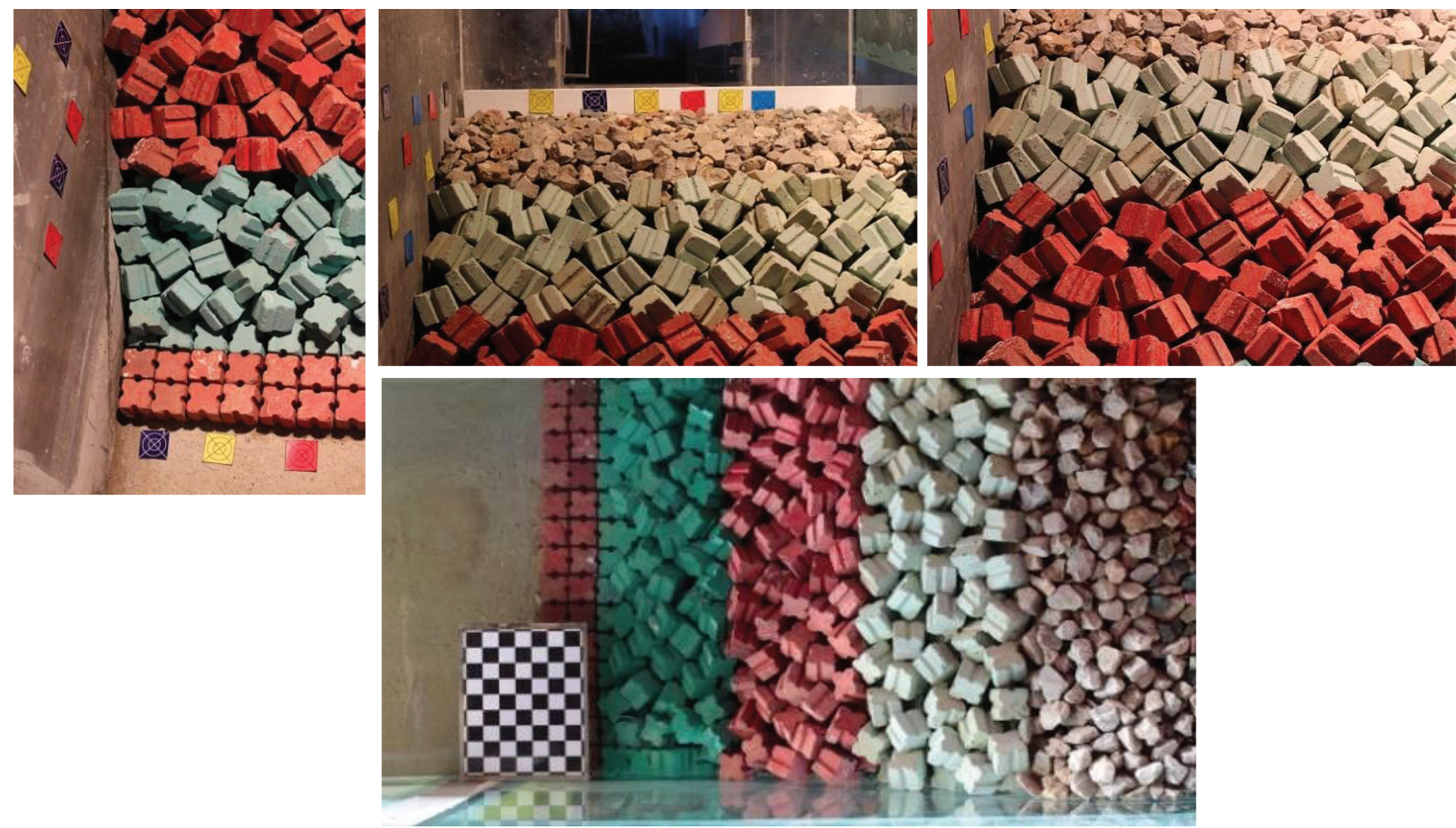

Fig. 6 Setup showing targets for reference coordinates.

(squares with edges of $3 \mathrm{~mm}$ ), which was stabilized above the water, for a water depth of $0.27 \mathrm{~m}$ (Figure 6). Only this last methodology enables surveying submerged scenes with light refraction corrections.

For the four methodologies, the resulting clouds of points were manipulated with an in-house MATLAB algorithm, the CloudCompare open-source software and the Golden Software Surfer ${ }^{\circledR}$ commercial software.

\section{THE 3D MODELLING METHODOLOGIES}

Photogrammetry is the science of making measurements from photographs; it is especially used to determine the exact positions of surface points. A special type, called "stereo photogrammetry", involves estimating the three-dimensional coordinates of points on an object employing measurements made in two or more photographic images taken simultaneously from different positions, where the common points are identified on each image. A large range of scanning techniques is also available for 3D modelling. Both techniques also enable the creation of cartographic products.

Nowadays, different commercial and open-source post-processing tools are available for a wide range of applications. The four previously mentioned methodologies will be described below to understand their pros and cons when applied to surveys of scale-model tests of rubble-mound breakwaters.

\section{1 Microsoft $^{\circledast}$ Kinect $^{\circledast}(\mathrm{MK})$}

The first methodology (MK) uses the Microsoft Kinect sensor (version 2.0), which is equipped with a depth sensor composed of an infrared projector and a monochrome complementary metal-oxide semiconductor (CMOS) sensor, which work together to survey the target surfaces in 3-D, regardless of the lighting. It is also equipped with a colour VGA video camera, which aids the detection of facial and other features through red, green and blue (RGB) colour components.

The acquisition of depth values by the Kinect is determined by the Time of Flight (ToF) method, which is based on the time elapsed between the transmission and reception of a signal. This method assumes that the speed of light is constant; it determines the time the signal takes to be returned to the sensor. ToF imaging refers to the process of measuring the depth of a scene by quantifying the changes that an emitted light signal encounters when it bounces back from objects in a scene (Castaneda \& Navab, 2011).

The Microsoft Kinect Fusion application uses the built-in Kinect cameras and projector to generate geometrically accurate 3D model meshes (Izadi et. Al, 2011) by applying a ToF technique with infrared light. This opens the possibility of rapidly and easily capturing data from objects or surfaces with a high reflectivity index and in low-visible lighting conditions. Using the sensor and the associated software, it is possible to capture objects at distances ranging from $0.5 \mathrm{~m}$ to $4.5 \mathrm{~m}$. According to tests performed by Fankhauser et al. (2015), the measured depth distortion was found to oscillate between $\pm 6 \mathrm{~mm}$. Its pixel dimension (Ground Sampling Distance) range is $1.4 \mathrm{~mm}$ at $0.5 \mathrm{~m}$ and $12 \mathrm{~mm}$ at $4.5 \mathrm{~m}$ (Rocha, 2017).

The 2,922,123 point cloud of the model was generated using the 3D mesh captured with the Kinect Fusion application and post-processing with CloudCompare. Due to the MATLAB matrix size limitations (used to further data processing), that number was reduced to 730,531 points, which allowed for a good manipulation of the point cloud.

The main advantages of the Kinect sensor are that only one (specific) camera is required; no manual depth computations are required; 3D scene geometry is acquired in real-time and there is reduced dependence on scene illumination and almost no dependence on surface texturing. 


\subsection{The Python Photogrammetry (PP) toolbox}

A second methodology (PP) uses the Python photogrammetry toolbox, which contains multiple processes to determine a 3D model of an area of study. During the experiments, 215 photos were obtained. This methodology, does not need external calibration, as the first process of the software, called "bundler", performs the camera calibrations and determines the position $(\mathrm{x}, \mathrm{y}, \mathrm{z})$ of the camera in each photo. This process is based on the common points found in a set of photos and on the knowledge of the camera's sensor size. After the determination of the camera position of each photo, it is necessary to perform another process called Patch Multi View Stereo (PMVS). This process has the set of photos as an input and the coordinates of the cameras in order to create a $3 \mathrm{D}$ point cloud based on the common points of each photo. It is possible to create a mesh based on the 3D cloud to which the set of photos will be associated in order to create a coloured 3D model with graphic information.

\subsection{MicMac (MM) software}

The third methodology used in this study is the MicMac methodology (MM). MicMac is an open-source photogrammetric suite developed at the French National Geographic Institute (IGN) and French National School for Geographic Sciences (ENSG). It can be used in a variety of 3D reconstruction scenarios, e.g., from small objects to large ones, such as buildings, breakwaters and dams, to produce point clouds and ortho-mosaics. From these, depth maps, digital surface models, etc., can be produced. Multi-Image Correspondences, Méthodes Automatiques de Corrélation (MicMac) is a powerful and accurate tool that has complicated processing as a drawback. It can be used either with terrestrial photos (terrestrial photogrammetry) or aerial photos (usually taken by digital cameras on UAV).

The survey with this methodology required the acquisition of 50 photos and the first steps of their processing are similar to the PP procedure. An important difference is that the camera calibration can be made by two different and independent ways. One of them is to use the photos of the survey (as PP does), the other one is using a set a photos taken with the propose of calibration, like photos of a corner of a structure or a similar scene with sufficient depth variation and obtained with the camera in several positions, as recommended by MicMac developers (MicMac, 2018).

After the orientation process (bundle adjustment), to determine the relative positions and orientations of the camera during the photo survey, the average residual was 0.45 pixel, being the worst value 0.93 pixel. According to the developers of Micmac, an excellent result is to have residuals less than 0.5 pixel. Residuals ranging between 0.5 and 1.0 are considered good results.

\subsection{In-house Software (IS) for the reconstruction of submerged scenes}

Finally, the fourth photogrammetric methodology (IS) consists of a scene-reconstruction software that rectifies the distortions introduced by the air-water interface, which means that it is possible to reconstruct both emerged and submerged scenes, without a need to empty the tank. This is clearly a major advantage of this methodology over the others.

The software package available (Ferreira, 2006) allows for a complete 3D reconstruction environment; it uses stereo image pairs taken by two fixed cameras as inputs. It consists of two distinct applications coded in MATLAB. The first application enables the camera's calibration, which is the process in which the geometric properties of a camera are estimated (Castro, 2015). It consists in identifying the parameters that describe the projective cameras and their position and orientation within the scene observed.

The calibration procedure consists of clicking the inside of four corners of the calibration pattern in a specific order (Figure 7 a). The first clicked corner specifies the origin of the pattern, while the second clicked corner defines the direction of the $\mathrm{X}$ axis. The second application has scene reconstruction as its objective and consists of estimating the three-dimensional coordinates from two different views of the same scene.

The basic principles of reconstruction are the stereo correspondence of homologous points in the stereo pairs as well as the triangulation for computing the position of the correspondence in the 3D space (Mattoccia, 2015).

The reconstruction procedure has a reconstruction file containing the matrixes for $\mathrm{x}, \mathrm{y}$ and $\mathrm{z}$ positions as an output, from which it is possible to extract the point cloud and subsequently the surface and pre-defined profiles (Figure $7 \mathrm{~b}$ ).

Despite being simple to use, this methodology requires a careful calibration procedure, since all the following procedures depend on it. Since surveys can be conducted for submerged scenes, light reflections in the water should be avoided in order to prevent distortions in the photogrammetric reconstruction.

During the present experiments, this methodology required 15 image pairs for camera calibration and an additional pair per survey. Its pixel dimensions (Ground Sampling Distance) are around $1.3 \mathrm{~mm}$, at a distance of $2.0 \mathrm{~m}$.

The point cloud number was limited to 89,377 to maintain a good compromise between the camera's highest resolution and the software code limitations. Considering an average-performance PC (e.g., 64-bit Intel Core i5@3.20 GHz, 6GB RAM), the computing time for extracting the point cloud and surface modelling for each survey was about twenty minutes for the camera calibration procedures and about
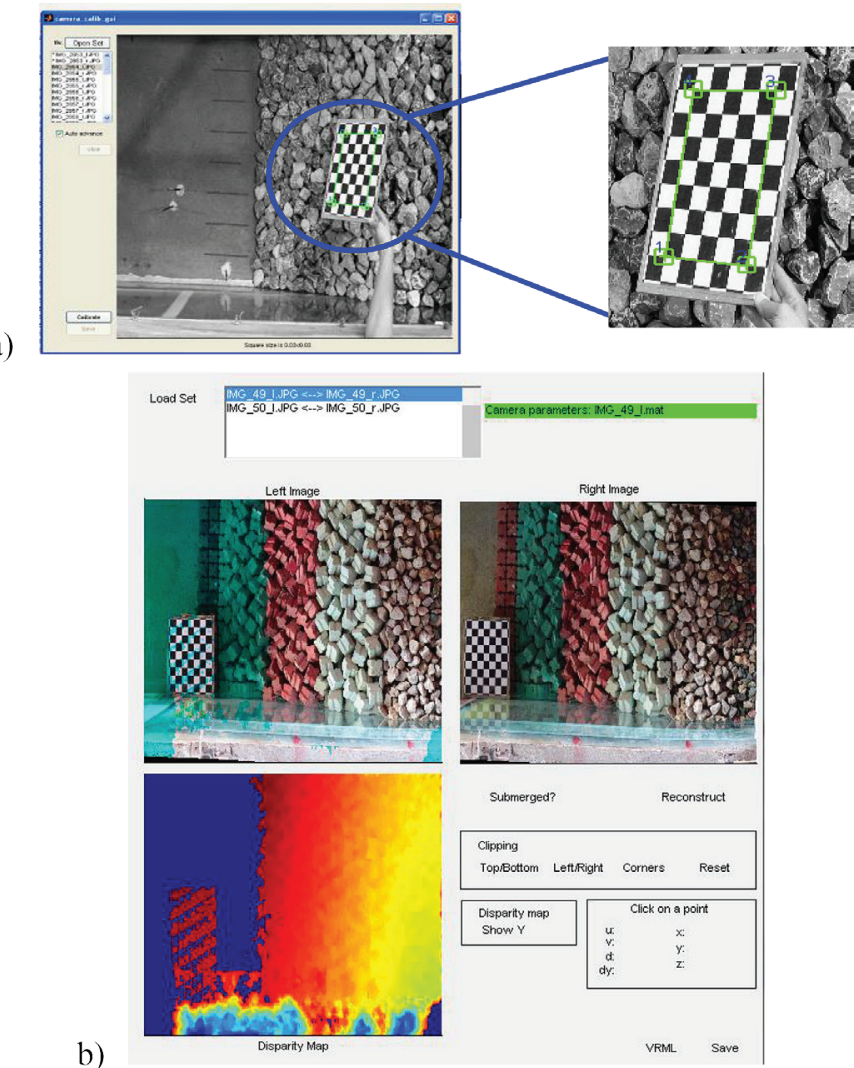

Fig. 7 Camera calibration (a) and scene reconstruction (b). 


\section{five minutes for the point cloud extraction.}

\section{RESULTS AND DISCUSSION}

In order to compare the different survey methodologies (MK, PP, MM and IS) applied to the physical scale models, a surface representation was conducted; it used a regular grid of $0.01 \mathrm{~m}$, as well as a profile extraction, which was approximately at the middle of the cross-section $(\mathrm{y}=0.4 \mathrm{~m})$.

Differences between the surveyed profiles and a theoretical profile based on the model's actual dimensions were computed.

Table 1 shows the average and maximum differences found for the surveys using the four methodologies. Figure 8 illustrates the difference of depth between the surveyed profile with the different methodologies and the theoretical profile (an imaginary, delimitating line, created upon the model's actual dimensions and the steepness of its slope - Figure 9). The theoretical profile was created based only on the armour slope, which was composed of Antifer cubes, in order to avoid some potential survey errors caused by the transition step between the armour layer, composed of cubes, and the upper part of the armor layer, composed of rocks.

The lower differences of the statistical parameters were found with the use of the IS methodology. The higher differences were found with the MM methodology.

Tab. 1 Differences in average and maximum depths from the four methodologies compared to a theoretical profile

\begin{tabular}{|c|c|c|c|c|}
\hline \multirow{2}{*}{} & \multicolumn{4}{|c|}{$\begin{array}{c}\text { Differences in depth from MK, PP, MM } \\
\text { or IS to a theoretical profile (m) }\end{array}$} \\
\cline { 2 - 5 } & MK & PP & MM & IS \\
\hline Average & 0.0337 & 0.0172 & 0.0352 & 0.0158 \\
\hline Max & 0.0617 & 0.0445 & 0.0709 & 0.0474 \\
\hline
\end{tabular}

Figure 10 illustrates the slope envelope obtained with each methodology. Note that for the MK methodology, the "y" coordinates are mirrored over the " $\mathrm{x}$ " axis, due to the sensor inversion.

a)

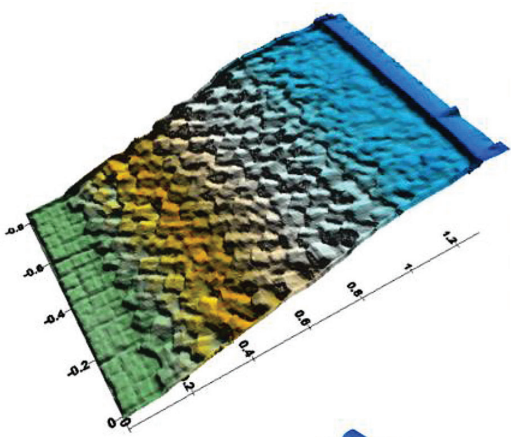

c)

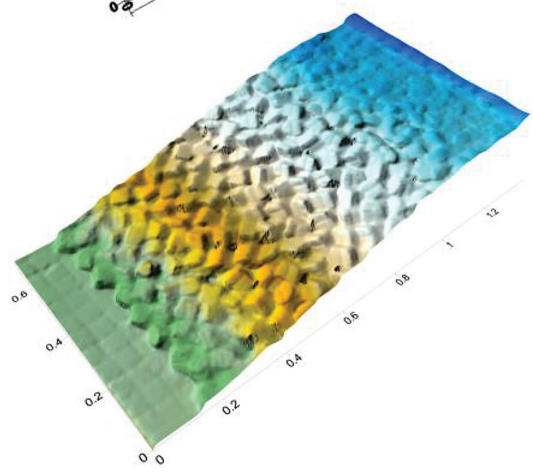

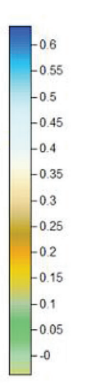

b)
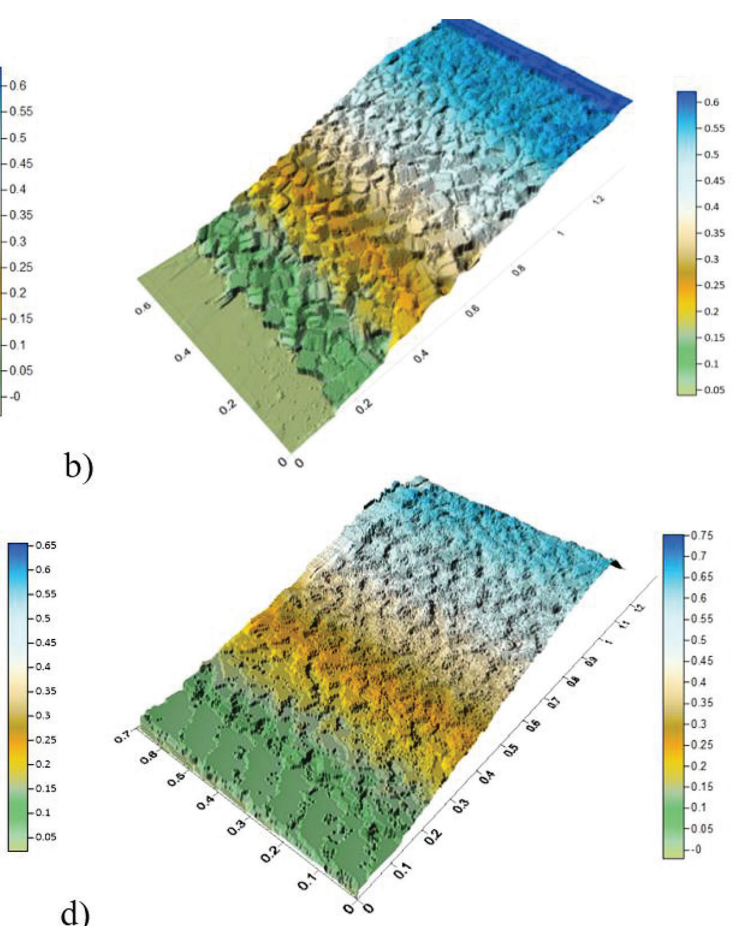

Table 2 shows some statistical parameters resulting from the quantification of the differences in depth between the grids obtained with the four methodologies.

Fig. 8 A comparison of the profiles obtained with the four methodologies.

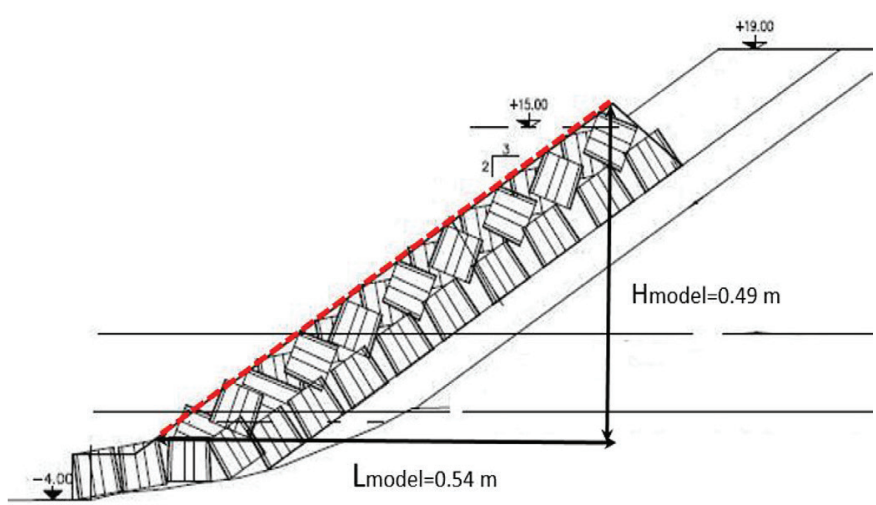

Fig. 9 Theoretical profile based on the model's actual dimensions.

Fig. 10 Model's envelopes obtained for: a) $M K$ b) MM c) PP and d) IS methodologies. 
Tab. 2 Statistic parameters resulting from the quantification of the depth differences between grids obtained with the four methodologies.

\begin{tabular}{|c|c|c|c|c|c|c|}
\hline & \multicolumn{6}{|c|}{ Compared grids/ Statistical parameters (m) } \\
\cline { 2 - 8 } & IS vs. MK & IS vs. MM & IS vs. PP & MK vs. MM & PP vs MK & PP vs. MM \\
\hline Maximum & 0.219 & 0.261 & 0.196 & 0.201 & 0.142 & 0.105 \\
\hline Mean & 0.068 & 0.074 & 0.051 & 0.045 & 0.018 & 0.024 \\
\hline Median & 0.071 & 0.081 & 0.054 & 0.041 & 0.019 & 0.021 \\
\hline
\end{tabular}
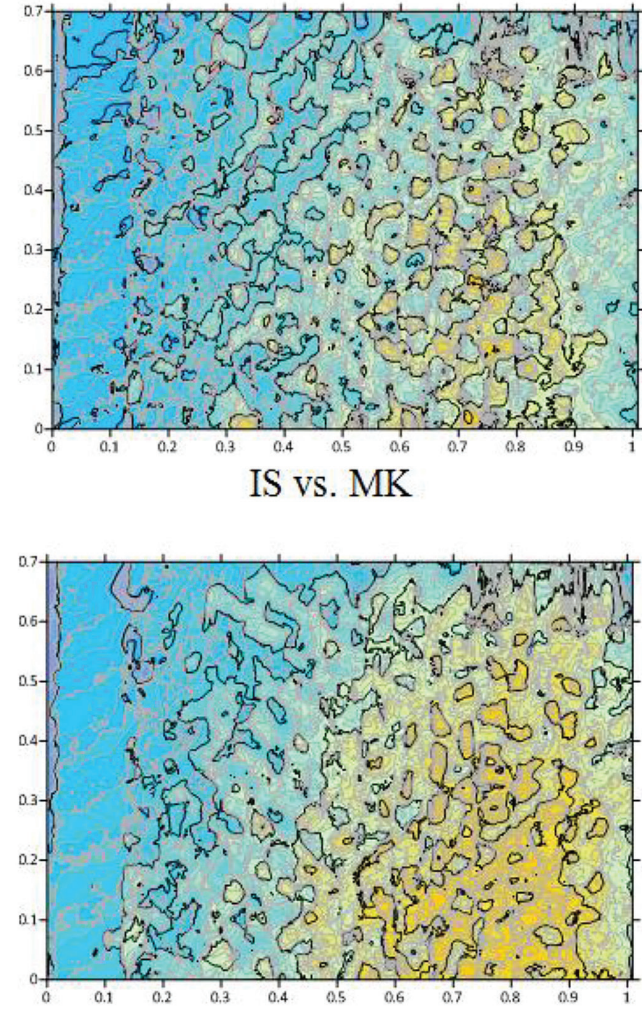

IS vs. PP

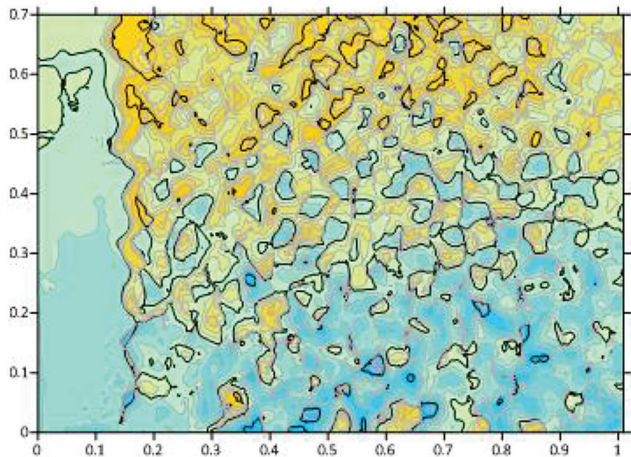

PP vs. MM

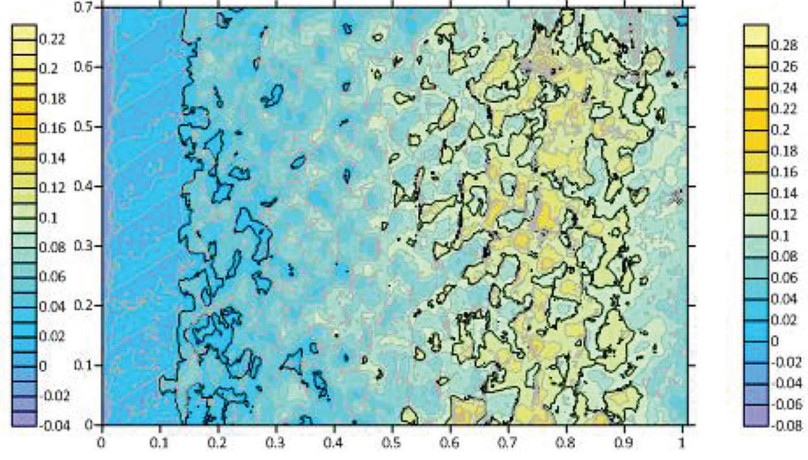

IS vs. MM
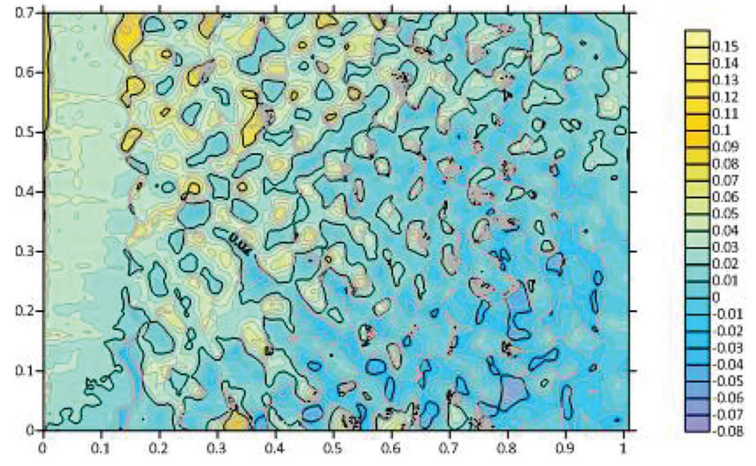

PP vs. MK
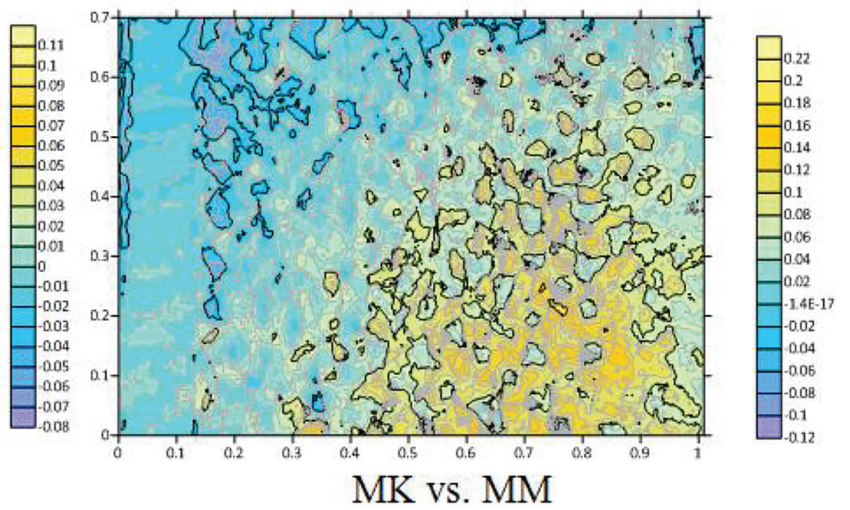

Fig. 11 Contours resulting from differences in depth between the grids obtained from the four methodologies.

The highest statistical parameter values obtained from the computed grid differences occurred between the surveys computed with the IS and MM methodologies, with a maximum of $0.26 \mathrm{~m}$, a mean of $0.074 \mathrm{~m}$ and a median of $0.081 \mathrm{~m}$. On the other hand, the lowest mean and median values were found between the PP and MK methodologies, i.e., $0.018 \mathrm{~m}$ and $0.019 \mathrm{~m}$ respectively. The lowest maximum difference $(0.105 \mathrm{~m})$ was found between the PP and MM methodologies.
Figure 11 illustrates the contours resulting from differences in depth between the grids obtained from the four methodologies.

Finally, Table 3 synthetizes the main characteristics of the four methodologies by quantifying the differences between the resolutions, processing complexity, specific application conditions, etc. 
Tab. 3 Main characteristics of the four methodologies.

\begin{tabular}{|c|c|c|c|c|}
\hline & & Met & dology & \\
\hline & $\begin{array}{l}\text { Using 3D scanning } \\
\text { with MK }\end{array}$ & $\begin{array}{l}\text { Photogrammetry } \\
\text { with MM }\end{array}$ & $\begin{array}{l}\text { Photogrammetry using } \\
\text { air/water interface } \\
\text { correction with IS }\end{array}$ & Photogrammetry with PP \\
\hline Number of photos/scan per survey & 1 & 50 & $\begin{array}{l}15 \text { pairs for camera } \\
\text { calibration }+1 \text { pair per } \\
\text { survey }\end{array}$ & 215 \\
\hline Photo processing complexity & None & High & Medium & Medium \\
\hline Points in the point cloud & 2.9 million & 2.6 million & 89377 & 166264 \\
\hline Point cloud post-processing complexity & Medium & Simple & Simple & Simple \\
\hline Computing time with an average performance PC & $20 \mathrm{~min}$ & Hours & $25 \mathrm{~min}$ & Hours \\
\hline Submerged sceneries allowed & No & No & Yes & No \\
\hline Expected precision based on previous experiments & Better than $2.5 \mathrm{~mm}$ & Not yet determined & Better than $2 \mathrm{~mm}$ & Not yet determined \\
\hline Light conditions dependency & Not very dependent & Dependent & Dependent & Dependent \\
\hline
\end{tabular}

\section{CONCLUSIONS}

This study describes four low-cost methodologies for surveying two-dimensional scale models of rubble mound breakwaters able to produce average errors below $3.5 \mathrm{~cm}$ when comparing with a theoretical profile.

The computing times of both the MK and IS methodologies are similar, despite some differences in the point cloud extraction and post-processing times. On the other hand, the surveys with the MM and PP methodologies require longer processing times.

The MK methodology demonstrated to be capable of providing the highest density of the point cloud, which resulted in higher quality images. Nevertheless, to speed up its post-processing procedures, the point cloud number had to be cut to a quarter of its number of points, which was nevertheless enough to obtain well-defined profiles. For this methodology and with regard to the degree of accuracy obtained by the present study $(0.0337 \mathrm{~m})$, we found it to be lower than the expected degree of accuracy based on previous experiments $(0.0025 \mathrm{~m})$.

Concerning the profile analysis, despite the poor resolution of the surface obtained with the IS methodology, which had an error of $0.016 \mathrm{~m}$ (which was higher than the expected accuracy of $0.002 \mathrm{~m}$ ), this methodology produced the lowest differences with the theoretical profile of the section being tested and also showed good agreement with the steepness of the slope.

However, since this was the only methodology where cameras were mounted in a structure and were placed perpendicularly to the flume, the relevance of the position of the cameras should be investigated. Therefore, additional surveys should be conducted (using the four methodologies) with cameras/sensors positioned in a normal plan, which seems to be beneficial to sloping scenerios and detrimental to prismatic volumes.

Concerning the mesh differences, higher values were found between the IS and MM methodologies. On the other hand, the lowest mesh differences were found between the PP and MK methodologies.

The accuracy associated with all the methodologies for profile surveying can be reduced by optimizing the camera position, facing the surveyed scene. Differences under $5 \mathrm{~mm}$ would be suitable for a profile survey of scale-model tests of rubble-mound breakwaters, by taking into account the dimensions of the surveyed scenes.

Finally, one can also conclude that all the methodologies required a significant amount of processing time. Nevertheless, for surveys of submerged scenes, the software developed for the reconstruction of submerged scenes (IS) is recommended. 


\section{REFERENCES}

Broderick, L.- Ahrens, J.P. (1982). Rip-rap stability scale effects. Technical Paper 82-3, U.S. Army Engineer Waterways Experiment Station, Coastal Engineering Research Center, Vicksburg, MS.

Castaneda, V.- Navab, N. (2011). Time-of-Flight and Kinect Imaging, lecture online, Munich Technical University.

http://campar.in.tum.de/twiki/pub/Chair/TeachingSs $11 \mathrm{Ki}-$ nect/2011-DSensors_LabCourse_Kinect.pdf

Castro, D.H. (2015). From images to point clouds practical considerations for three-dimensional computer vision. Academic dissertation. University of Oulu, Finland.

Fankhauser, P. - Bloesch, M. - Rodriguez, D. - Kaestner, R. Hutter, M. - Siegwart, R. (2015). Kinect v2 for mobile robot navigation: Evaluation and modeling. Proceedings of the 17th International Conference on Advanced Robotics, ICAR 2015, pp. 388-394.

Ferreira, R. (2006). Reconstruction of a Submerged Model Breakwater and Interface Estimation. Master's degree thesis. Instituto Superior Técnico, Lisbon, Portugal.

Hofland, B. - Disco, M. - Van Gent, M.R.A. (2014). Damage characterization of rubble mound roundheads. In: CoastLab2014. Varna, Bulgaria.

Hofland, B. Rosa-Santos - P., Taveira-Pinto, F. - Almeida, E. - Lemos, R. - Mendonça, A.- Fortes, C.J.E.M. (2017). Measuring damage in physical model tests of rubble mounds. Proceedings of ICE Breakwaters 2017, 5 - 7 Sept. 2017 - Liverpool Waterfront, UK, 2017
Izadi, S. - Kim, D. - Hilliges, O. - Molyneaux, D. - Newcombe, R. - Kohli, P. - Shotton, J. - Hodges, S. - Freeman, D. - Davison, A, - Fitzgibbon, A. (2011). KinectFusion: Realtime $3 D$ Reconstruction and Interaction Using a Moving Depth Camera. UIST'11, Oct. 1619, 2011, Santa Barbara, CA, USA.

Mattoccia, S. (2015). Stereo Vision: Algorithms and Applications. Department of Computer Science (DISI), University of Bologna.

Pierrot-Deseilligny, M. - Rupnik, E. - Girod, L. - Belvaux, J. Maillet, G. - Deveau, M. - Choqueux, G. (2018). MicMac, Apero, Pastis and Other Beverages in a Nutshell. MicMac user's manual, Ecole Nationnale des Sciences Géographiques

https://raw.githubusercontent.com/micmacIGN/Documentation/master/DocMicMac.pdf

Rocha, C. (2107). Monitorização dos modelos de quebra-mares com o sensor Microsoft Kinect V2. Tese de Mestrado. Faculdade de Ciências de Lisboa.

Van der Meer, J. W. (1988). Stability of Cubes, Tetrapodes and Accropodes. Proceedings of the Breakwaters ' 88 Conference, Design of Breakwaters, Institution of Civil Engineers, Thomas Telford, London, UK, pp. 71-80. 